\title{
$P$ ve $n$ katkılı grafen elektrotlara asimetrik bağlı güçlü korelasyonlu bir kuantum noktacığında gerçek zamanlı elektron dinamikleri
}

\author{
Ali İhsan GÖKER* \\ Bilecik Şeyh Edebali Üniversitesi, Fen Edebiyat Fakültesi, Fizik Bölümü, 11210, Gülümbe Kampüsü, Bilecik \\ Geliş Tarihi (Recived Date): 19.08.2017 \\ Kabul Tarihi (Accepted Date): 01.11.2017
}

\begin{abstract}
Özet
Kesişmeme yaklaşımını kullanarak $p$ ve $n$ katkılı grafen elektrotlara asimetrik olarak bağlanmıı̧ güçlü korelasyonlu bir kuantum noktacığı boyunca kuantum taşınmasını araştırdık. Kuantum noktacı̆̆ı bir kapı voltajı yoluyla aniden elektrotların Fermi düzeyine yakın bir noktaya getirildiğinde meydana gelen iletkenliğin karmaşık dalgalanmalar gösterdiğini bulduk. Bu dalgalanmaların ortam sıcaklı̆̆ Kondo sıcaklı̆̆ının üstüne çıktığında ve asimetri faktörü azaldığında giderek sönümlendiğini tespit ettik. Gözlemlenen dalgalanmaları Fourier dönüşümmü yoluyla analizi sonucunda, titreşimlerin $n$ katkllı grafende Dirac noktası ve bant kenarının Fermi düzeyine uzaklı̆̆lyla orantıll iki farklı frekansta gerçekleşirken $p$ katkll grafende bant kenarının Fermi düzeyine uzakllğıyla orantıll sadece tek bir titreşim frekansı olduğunu bulduk. Вu durumu Fermi düzeyindeki gelişen Kondo rezonansinın elektrotların durumlar yoğunluğundaki van Hove tekillikleriyle dinamik girişimini öneren bir mikroskopik teoriye bağladık.
\end{abstract}

Anahtar Sözcükler: Grafen, Kondo etkisi, kuantum noktacı̆̆ı.

\section{Real time electron dynamics in a strongly correlated quantum dot asymmetrically coupled to $\mathrm{p}$ and $\mathrm{n}$ doped graphene electrodes}

\begin{abstract}
We investigated the quantum transport through a strongly correlated quantum dot asymmetrically coupled to $p$ and $n$ doped graphene electrodes invoking the non-crossing approximation. We found that the conductance exhibits complex fluctuations when the dot level is abruptly moved to a position close to the Fermi level of the electrodes via a gate voltage. We determined that these fluctuations get gradually suppressed when the ambient temperature starts to exceed the Kondo temperature and the asymmetry factor decreases. As a result of the analysis of these fluctuations via the Fourier transform, we found that while the oscillations take place in two distinct frequencies which are proportional to the distance of the Dirac point and the band edge to the Fermi level for the $n$ doped graphene, there exists only one oscillation frequency proportional to the distance of the band edge to the Fermi level. We attributed this situation to a microscopic theory which suggests the dynamical interference of the developing Kondo resonance at the Fermi level with the van Hove singularities at the density of states of the electrodes.
\end{abstract}

Keywords: Graphene, Kondo effect, quantum dot.

\footnotetext{
*Ali İhsan GÖKER, aihsan.goker@ bilecik.edu.tr, http://orcid.org/0000-0001-8645-4617
} 


\section{Giriş}

Tek elektron tranzistörlerinin ani açılıp kapanma durumundaki davranışlarının tespit edilebilmesi giderek önem kazanmaktadır çünkü günümüzde elektronik cihazlarda kullanılan geleneksel metaloksit yariiletken alan etkili tranzistörlerin giderek küçülerek üretimlerinin zorlaşmasından dolayı tek elektron tranzistörlerin gelecekte yaygınlaşması beklenen organik elektronik cihazların yapıtaşlarını oluşturacağı düşünülmektedir. Daha önce bu cihazlarda kapı voltajının adım şeklindeki ani değişiminden kaynaklanan pertürbasyonların etkileri incelenmiş [1-3] ve bu pertürbasyonun sonucu oluşan zamana bağlı akımın değişik zaman ölçekleri gösterdiği tespit edilmiştir [4, 5]. İlk kısa zaman ölçeği kuantum noktacığının kesik düzeyinin ani yer değiştirmesinden dolayı bu düzeyin etrafındaki Breit-Wigner rezonansının yer değiştirmesiyle karakterize edilirken, bundan sonraki uzun zaman ölçeği oldukça hassas bir çok parçacık etkisi olan Kondo rezonansının elektrotların Fermi düzeyinde gelişmesiyle ilişkilidir. Daha sonraki araştırmalar kuantum noktacığının elektrotlarla asimetrik eşleşmesinin Kondo rezonansı ile elektrotların durumlar yoğunluğundaki van Hove tekillikleri adı verilen süreksizlikler ile girişim doğuracağını ortaya atmıştır [6].

Grafen, $s p^{2}$ hibritleşmiş karbon atomlarından oluşmuş tek bir katmanın hegzagonal balpeteği kafesi şeklinde kristalleşmiş halidir. Aşırı yüksek taşıyıcı mobilitesine sahip ilk gerçek anlamda iki boyutlu kristal malzeme olması ve Dirac noktası etrafında sıfır bant açıklığıyla lineer dispersiyon gösteren kendine özgü elektronik yapısından dolayı ilk kez taşınma deneyleri ile izole ve karakterize edilmesinden beri [7] yoğun bilimsel ilgi görmektedir. Grafen, kendine özgü optik özellikleri olan şeffaf bir iletken [8], ölçülen en yüksek elastisiteye sahip çok güçlü bir malzeme [9] ve üstün termal bir iletkendir [10]. Dolayısıyla, hem temel araştırmalar hem de teknolojik uygulamalar için uygun bir platform oluşturmaktadır.

Elektrotların arasına yerleştirilmiş kuantum noktacıkları ve moleküllerde elektronlar arası Coulomb etkileşiminin gözönüne alınması için ilk girişim Anderson Hamiltonyan'idir [11]. Bu modelde, Kondo ölçeği adı verilen bir sıcaklığın altında farklı spine sahip iki elektronun aynı anda tünellemesi sonucunda kuantum noktacığının kesik enerji düzeyinde spin dönmesi olayları meydana gelir. Bunun sonucunda elektrotların Fermi düzeyinde keskin bir rezonans ortaya çıkar. Kondo rezonansı adlı bu hayali parçacık rezonansı Coulomb blokajı altındaki bu devreden akım geçmesine yol açar.
Anderson Hamiltonyan'ını çözmek için kullanılan en yaygin metot ilk kez Kadanoff ve Baym tarafından geliştirilen [12] ve daha sonra lineer olmayan tepki problemlerine genelleştirilen [13] denge dışı Green fonksiyonlarıdır. Denge dışı Green fonksiyonlarının elde edilebilmesi için çözülmesi gereken Dyson denklemlerinde elektronun kendisiyle etkileşiminden doğan enerjisinin elde edilebilmesi için Anderson modelinin köle bozonlar yardımıyla dönüşümü öne sürülmüştür [14]. Bu yaklaşımı kullanarak çözümü ilk elde eden ise Nordlander ve çalışma arkadaşları olmuştur [15-16].

$\mathrm{Bu}$ makalede ilk kez $\mathrm{p}$ ve $\mathrm{n}$ katkılı grafen elektrotlarla asimetrik olarak eşleşmiş güçlü Coulomb etkileşmesine sahip bir kuantum noktacığının kesik enerji düzeyi bir kapı voltajı yoluyla aniden elektrotların Fermi düzeyine yakın bir seviyeye çıkarıldığında Kondo zaman ölçeğinde devreden geçen akımı değişik parametrelerle inceleyip elde edilen sonuçları yorumlamaya çalışacağız.

\section{Materyal ve metot}

Grafen elektrotlara bağlı Coulomb etkileşimine sahip bir kuantum noktacığını $\varepsilon_{d}$ enerjisine sahip ve tünel bariyerler yoluyla elektrotlarla eşleşmiş tek bir spin dejenere kesik düzeyle göstereceğiz. Buna karşılık gelen Hamiltonyan

$$
\begin{aligned}
& \mathrm{H}(\mathrm{t})=\sum_{\sigma} \varepsilon_{\mathrm{d}}(\mathrm{t}) \mathrm{c}_{\sigma}^{\mathrm{t}} \mathrm{c}_{\sigma}+\sum_{\mathrm{k} \alpha \sigma} \varepsilon_{\mathrm{k}} \mathrm{c}_{\mathrm{k} \alpha \sigma}^{\mathrm{t}} \mathrm{c}_{\mathrm{k} \alpha \sigma}+ \\
& \frac{1}{2} \sum \mathrm{U} \mathrm{c}_{\sigma}^{\mathrm{t}} \mathrm{c}_{\sigma} \mathrm{c}_{\sigma^{\prime}}^{\mathrm{t}} \mathrm{c}_{\sigma^{\prime}}+\sum_{\mathrm{k} \alpha \sigma}\left[\mathrm{V}_{\alpha}\left(\varepsilon_{\mathrm{k} \alpha}\right) \mathrm{c}_{\mathrm{k} \alpha \sigma}^{\mathrm{t}} \mathrm{c}_{\sigma}+\text { h.c. }\right]
\end{aligned}
$$

şeklinde yazılabilir. Burada $c_{\sigma}^{t}\left(c_{\sigma}\right) v e \quad c_{k \alpha \sigma}^{t}\left(c_{k \alpha \sigma}\right)$ sırayla kuantum noktacığındaki elektrotlardaki $(\alpha=\mathrm{L}, \mathrm{R}) \quad \sigma$ spinine sahip bir elektronu oluşturma (yok etme) operatörleridir. $\mathrm{V}_{\alpha}$ ise elektrotlar ve kuantum noktacığı arasındaki elektron atlama genliğidir. Coulomb etkileşim şiddeti $U$ ile gösterilmekte olup kuantum noktacığındaki hapsedilmeden dolayı kesik enerji düzeyinde aynı anda iki elektronun barınmasını önleyecek kadar güçlü olduğu varsayılacaktır. $\mathrm{Bu}$ makalede $\hbar=\mathrm{k}_{\mathrm{B}}=\mathrm{e}=1$ olacak şekilde atomik birimleri kullanacağız.

Güçlü Coulomb etkileşiminin altından kalkabilmek için yardımcı bozon metodunu kullanacağız. Bu metotta elektron operatörleri yalancı fermiyon ve kütlesiz bozon operatörleri yardımıyla

$c_{\sigma}=b^{t} f_{\sigma}$

olarak 
$Q=b^{t} b+\sum_{\sigma} f_{\sigma}^{t} f_{\sigma}=1$

şartına tabi olarak yeniden yazılabilir. $\mathrm{Bu}$ yeni operatörler cinsinden çift zamanlı yalancı fermiyon ve köle bozon küçüktür Green fonksiyonları $\mathrm{G}^{<}\left(\mathrm{t}, \mathrm{t}^{\prime}\right)=\left\langle\mathrm{f}_{\sigma}^{\mathrm{t}}\left(\mathrm{t}^{\prime}\right) \mathrm{f}_{\sigma}(\mathrm{t})\right\rangle \quad$ ve $\quad \mathrm{B}^{<}\left(\mathrm{t}, \mathrm{t}^{\prime}\right)=\left\langle\mathrm{b}^{\mathrm{t}}\left(\mathrm{t}^{\prime}\right) \mathrm{b}(\mathrm{t})\right\rangle \quad$ olarak yazılırken çift zamanlı yalancı fermiyon ve köle bozon küçüktür Green fonksiyonları ise $G^{>}\left(t, t^{\prime}\right)=\left\langle f_{\sigma}(t) f^{t}\left(t^{\prime}\right)\right\rangle \quad$ ve $\quad B^{>}\left(t, t^{\prime}\right)=\left\langle b(t) b^{t}\left(t^{\prime}\right)\right\rangle \quad$ şeklinde ifade edilir. Bunlar cinsinden geri yalanc1 fermiyon ve köle bozon Green fonksiyonları ise,

$$
\begin{aligned}
& G^{R}\left(t, t^{\prime}\right)=-i \theta\left(t-t^{\prime}\right)\left[G^{>}\left(t, t^{\prime}\right)+G^{<}\left(t, t^{\prime}\right)\right] \\
& B^{R}\left(t, t^{\prime}\right)=-i \theta\left(t-t^{\prime}\right)\left[B^{>}\left(t, t^{\prime}\right)-B^{<}\left(t, t^{\prime}\right)\right]
\end{aligned}
$$

olarak verilir.

$\mathrm{Bu} \quad$ ifadelerde $\quad \mathrm{B}^{\mathrm{R}}\left(\mathrm{t}, \mathrm{t}^{\prime}\right)=-\mathrm{i} \theta\left(\mathrm{t}-\mathrm{t}^{\prime}\right) \mathrm{b}\left(\mathrm{t}, \mathrm{t}^{\prime}\right) \quad \mathrm{ve}$ $G^{R}\left(t, t^{\prime}\right)=-i \theta\left(t-t^{\prime}\right) g\left(t, t^{\prime}\right) \quad$ kısaltmalarını kullanabiliriz. $\mathrm{Bu}$ şekilde ifade edilen geri yalancı fermiyon ve köle bozon Green fonksiyonları

$$
\begin{aligned}
& {\left[\frac{\partial}{\partial \mathrm{t}}+\mathrm{i} \varepsilon_{\mathrm{d}}\right] \mathrm{g}\left(\mathrm{t}, \mathrm{t}^{\prime}\right)=-\int_{\mathrm{t}^{\prime}}^{\mathrm{t}} \mathrm{dt}_{1} \mathrm{~K}^{>}\left(\mathrm{t}, \mathrm{t}_{1}\right) \mathrm{b}\left(\mathrm{t}, \mathrm{t}_{1}\right) \mathrm{g}\left(\mathrm{t}_{1}, \mathrm{t}^{\prime}\right)} \\
& \frac{\partial}{\partial t} b\left(t, t^{\prime}\right)=-\int_{t^{\prime}}^{t} d t_{1} K^{<}\left(t_{1}, t\right) g\left(t, t_{1}\right) b\left(t_{1}, t^{\prime}\right)
\end{aligned}
$$

ile verilen Dyson denklemlerinin çözümüyle bulunur [17]. Bu denklemlerdeki kerneller yalancı fermiyon ve köle bozon kendi enerjilerinin kesişmeme yaklaşımı yardımıyla yazılmasıyla elde edilir ve

$$
\begin{aligned}
& \mathrm{K}^{<}\left(\mathrm{t}, \mathrm{t}^{\prime}\right)=\bar{\Gamma}_{\text {tot }} \int_{-\mathrm{D}}^{\mathrm{D}} \frac{\mathrm{d} \varepsilon}{2 \pi} \rho(\varepsilon) \frac{\mathrm{e}^{\mathrm{i} \varepsilon\left(\mathrm{t}-\mathrm{t}^{\prime}\right)}}{1+\mathrm{e}^{\beta \varepsilon}} \\
& \mathrm{K}^{>}\left(\mathrm{t}, \mathrm{t}^{\prime}\right)=\bar{\Gamma}_{\text {tot }} \int_{-\mathrm{D}}^{\mathrm{D}} \frac{\mathrm{d} \varepsilon}{2 \pi} \rho(\varepsilon) \frac{\mathrm{e}^{\beta \varepsilon+\mathrm{i} \varepsilon\left(\mathrm{t}-\mathrm{t}^{\prime}\right)}}{1+\mathrm{e}^{\beta \varepsilon}}
\end{aligned}
$$

ile verilir. Burada $\beta$ ortam sıcaklığının tersi olup $\rho(\varepsilon)$ yarım bant genişliği $D$ olan elektrotların durumlar yoğunluğunu göstermektedir. Sol ve sağ elektrot aynı olduğu için $\rho_{L(R)}(\varepsilon)=\rho(\varepsilon)$ olarak alınabilir. Tünelleme genliğinin enerji bağımlılığını ihmal ederek $V_{L(R)}(\varepsilon)=V_{L(R)}\left(\varepsilon_{F}\right)$ olarak kabul edeceğiz. Kuantum noktacığının elektrotlarla eşleşme fonksiyonu $\Gamma_{L(R)}(\varepsilon)=\bar{\Gamma}_{L(R)} \rho(\varepsilon)$ şeklinde parametrize edilebilir. $\quad \mathrm{Bu}$ ifadede $\quad \bar{\Gamma}_{\mathrm{L}(\mathrm{R})}$ sabit olup $\bar{\Gamma}_{L(R)}=2 \pi\left|V_{L(R)}\left(\varepsilon_{F}\right)\right|^{2} \quad$ şeklinde ifade edilir. Bu sabitler cinsinden $\quad \Gamma_{\text {tot }}=\bar{\Gamma}_{\text {tot }} \rho\left(\varepsilon_{\mathrm{F}}\right)=\left(\bar{\Gamma}_{\mathrm{L}}+\bar{\Gamma}_{\mathrm{R}}\right) \rho\left(\varepsilon_{\mathrm{F}}\right)$ sonucuna ulaşırız. Asimetri faktörü olarak $\eta=\frac{\bar{\Gamma}_{L}}{\bar{\Gamma}_{L}+\bar{\Gamma}_{R}}$ tanımını kullanacağız.

Kuantum noktacığının kesik enerji düzeyi $\varepsilon_{d}$ elektrotların Fermi düzeyinin yeterince altında olduğu durumlarda kuantum noktacı̆̆ının durumlar yoğunluğu iki rezonanstan oluşur. Bunların birincisi $\varepsilon_{d}$ etrafında oluşan ve genişliği $\Gamma_{t o t}$ kadar olan BreitWigner rezonansıdır. Bunun altında yatan kuantum noktacığ 1 ve elektrotlardaki elektronlar arasındaki tünellemeden kaynaklanan hibritleşmedir. Diğer rezonans ise elektrotların Fermi düzeyinde oluşan ve genişliği

$$
\mathrm{T}_{\mathrm{K}} \approx \frac{\sqrt{\mathrm{D} \Gamma_{\mathrm{tot}}}}{2} \exp \left(-\frac{\pi\left|\varepsilon_{\mathrm{d}}\right|}{\Gamma_{\mathrm{tot}}}\right)
$$

ile verilen Kondo sıcaklığı mertebesinde olan Kondo rezonansidır [18]. $\mathrm{Bu}$ rezonans ortam sicaklı̆̆ına oldukça duyarlı olup, ortam sıcaklığı $T_{K}$ 'nın üstüne çıktığı durumda giderek yok olmaya başlar. Kondo rezonansına yol açan ise iki elektronun aynı anda tünellemesiyle kuantum noktacı̆̆ındaki yukarı veya aşağı spine sahip bir elektronun yerini elektrotlardan gelen zit spine sahip başka bir elektronun almasıdır. Buna spin dönmesi olayı adı verilir.

Geri Green fonksiyonları 6. ve 7. denklemler çözülerek bulunduktan sonra küçüktür Green fonksiyonları

$$
\begin{aligned}
& {\left[\frac{\partial}{\partial t}+i \varepsilon_{d}\right] G^{<}\left(t, t^{\prime}\right)=\int_{-\infty}^{t^{\prime}} d t_{1} K^{<}\left(t, t_{1}\right) B^{<}\left(t, t_{1}\right) g\left(t_{1}, t^{\prime}\right)} \\
& -\int_{-\infty}^{t} d t_{1} K^{>}\left(t, t_{1}\right) b\left(t, t_{1}\right) G^{<}\left(t_{1}, t^{\prime}\right) \\
& \frac{\partial}{\partial t} B^{<}\left(t, t^{\prime}\right)=\int_{-\infty}^{t^{\prime}} d t_{1} K^{>}\left(t_{1}, t\right) G^{<}\left(t, t_{1}\right) b\left(t_{1}, t^{\prime}\right) \\
& -\int_{-\infty}^{t} d t_{1} K^{<}\left(t_{1}, t\right) g\left(t, t_{1}\right) B^{<}\left(t_{1}, t^{\prime}\right)
\end{aligned}
$$

Dyson denklemleri çözülerek elde edilir [17]. Bu çözüm prosedüründe Green fonksiyonlarının değerleri kare bir matriste saklanır. Diyagonal elemanlar eşit zaman değerlerine karşılık gelir. Kare matris diyagonal yönde ilerletilerek zamana göre değişim elde edilir [19]. Bu ş̧ekilde Green fonksiyonları elde edildikten sonra devreden geçen net elektrik akım $I(t)=I_{L}(t)-I_{R}(t)$ ile verilir. Burada $I_{L}(t)\left(I_{R}(t)\right)$ sol (sağ) elektrottan kuantum noktacığına akan akımdır. Net akım Green fonksiyonları yardımıla [6] 


$$
\begin{aligned}
& \mathrm{I}(\mathrm{t})=-2\left(\bar{\Gamma}_{\mathrm{L}}-\bar{\Gamma}_{\mathrm{R}}\right) \operatorname{Re}\left[\int_{-\infty}^{\mathrm{t}} \mathrm{dt}_{1} \mathrm{G}^{<}\left(\mathrm{t}, \mathrm{t}_{1}\right) \mathrm{b}\left(\mathrm{t}_{1}, \mathrm{t}\right) \mathrm{h}\left(\mathrm{t}-\mathrm{t}_{1}\right)\right] \\
& +2 \bar{\Gamma}_{\mathrm{L}} \operatorname{Re}\left\{\int_{-\infty}^{\mathrm{t}} \mathrm{dt}_{1}\left[\mathrm{G}^{<}\left(\mathrm{t}, \mathrm{t}_{1}\right) \mathrm{b}\left(\mathrm{t}_{1}, \mathrm{t}\right)+\mathrm{g}\left(\mathrm{t}, \mathrm{t}_{1}\right) \mathrm{B}^{<}\left(\mathrm{t}_{1}, \mathrm{t}\right)\right] \mathrm{f}_{\mathrm{L}}\left(\mathrm{t}-\mathrm{t}_{1}\right)\right\} \\
& -2 \bar{\Gamma}_{\mathrm{R}} \operatorname{Re}\left\{\int_{-\infty}^{\mathrm{t}} \mathrm{dt}_{1}\left[\mathrm{G}^{<}\left(\mathrm{t}, \mathrm{t}_{1}\right) \mathrm{b}\left(\mathrm{t}_{1}, \mathrm{t}\right)+\mathrm{g}\left(\mathrm{t}, \mathrm{t}_{1}\right) \mathrm{B}^{<}\left(\mathrm{t}_{1}, \mathrm{t}\right)\right] \mathrm{f}_{\mathrm{R}}\left(\mathrm{t}-\mathrm{t}_{1}\right)\right\}
\end{aligned}
$$

$$
\begin{aligned}
& \text { halinde } \\
& \mathrm{h}\left(\mathrm{t}-\mathrm{t}_{1}\right)=\int_{-\mathrm{D}}^{\mathrm{D}} \frac{\mathrm{d} \varepsilon}{2 \pi} \rho(\varepsilon) \mathrm{e}^{\mathrm{i} \varepsilon\left(\mathrm{t}-\mathrm{t}_{1}\right)} i \text { iken } \\
& \mathrm{f}_{\mathrm{L}}\left(\mathrm{t}-\mathrm{t}_{1}\right)=\int_{-\mathrm{D}}^{\mathrm{D}} \frac{\mathrm{d} \varepsilon}{2 \pi} \rho(\varepsilon) \frac{\mathrm{e}^{\mathrm{i} \varepsilon\left(\mathrm{t}-\mathrm{t}_{1}\right)}}{1+\mathrm{e}^{\beta\left[\varepsilon-\frac{\mathrm{v}}{2}\right]}} \\
& \mathrm{f}_{\mathrm{R}}\left(\mathrm{t}-\mathrm{t}_{1}\right)=\int_{-\mathrm{D}}^{\mathrm{D}} \frac{\mathrm{d} \varepsilon}{2 \pi} \rho(\varepsilon) \frac{\mathrm{e}^{\mathrm{i} \varepsilon\left(\mathrm{t}-\mathrm{t}_{1}\right)}}{\left.1+\mathrm{e}^{\beta\left[\varepsilon+\frac{\mathrm{V}}{2}\right]}\right]}
\end{aligned}
$$$$
\mathrm{Bu}
$$
denklemde

olarak verilir. Burada $V$ elektrotlar arasında uygulanan voltaj değeridir

\section{Sonuçlar ve tartışma}

Bu kısımda grafen elektrotlara bağlı bir kuantum noktacığından oluşan bir sistemde kuantum noktacığın kesik enerji düzeyi aniden $\varepsilon_{\mathrm{d} 1}=-4 \Gamma_{\text {tot }}$ seviyesinden $\quad \varepsilon_{\mathrm{d} 2}=-2 \Gamma_{\text {tot }}$ seviyesine $\mathrm{t}=0$ anında çıkarıldıktan sonra devrede oluşan anlık iletkenliği inceleyeceğiz. Hesaplamalarımızda $\Gamma_{\text {tot }}=0.8 \mathrm{eV}$ ve $D=9 \Gamma_{\text {tot }}$ alacağız. Bu parametrelerle 10. denkleme göre ilk seviye için $T_{K}$ neredeyse sıfır iken son seviyede $\mathrm{T}_{\mathrm{K}} \approx 0.002 \Gamma_{\text {tot }}$ olmaktadır. Yani bu ani hareket Kondo rezonansının olmadığı bir durumdan $\left(\mathrm{T}>\mathrm{T}_{\mathrm{K}}\right.$ ), Kondo rezonansının zamanla geliştiği bir duruma geçişe karşılık gelmektedir. Dolayısıyla, kuantum noktacığı Coulomb blokajında olduğu için ilk durumda iletkenlik sıfirdır. Son durumda ise asimetrik bağlanmadan dolayı, ilk ani bir yükselişten sonra zamana bağlı olarak durgunluk düzeyine doğru yavaş bir iniş gözlenir.

Hesaplamalarımızda kullanacağımız elektrotlar değişik katkı seviyesindeki grafenden oluşmaktadır. Kullanacağımız üç farklı grafen elektrodun durumlar yoğunluğu Şekil 1'de gösterilmiştir. Burada, panel a ve b iki farklı katkı seviyesindeki n katkılı grafen elektrodun durumlar yoğunluğunu gösterirken, panel c ise p katkılı grafen elektrodun durumlar yoğunluğunu temsil etmektedir. Bütün panellerde Dirac noktasının konumu yarım bant uzunluğu olan $D$ cinsinden de işaretlenmiştir. Fermi düzeyi Dirac noktasının üstünde yer aldığ $\breve{i c ̧ i n}$ a ve $b$ panelleri $n$ katkıyı, Fermi düzeyi Dirac noktasının altında yer aldığı c paneli p katkıyı temsil etmektedir. Panel a ve b'deki n katkılı elektrotların durumlar yoğunluğu açık olarak

$$
\begin{aligned}
& \rho(\varepsilon)=\left\{\begin{array}{cc}
2.17 \varepsilon+0.723 \mathrm{D} & -\frac{\mathrm{D}}{3}<\varepsilon<\mathrm{D} \\
-2.17 \varepsilon-0.732 \mathrm{D} & -\mathrm{D}<\varepsilon \leq-\frac{\mathrm{D}}{3}
\end{array}\right. \\
& \text { ve } \\
& \rho(\varepsilon)=\left\{\begin{array}{cc}
2.17 \varepsilon+1.085 \mathrm{D} & -\frac{\mathrm{D}}{2}<\varepsilon<\mathrm{D} \\
-2.17 \varepsilon-1.085 \mathrm{D} & -\mathrm{D}<\varepsilon \leq-\frac{\mathrm{D}}{2}
\end{array}\right.
\end{aligned}
$$

şeklinde verilirken, panel c'deki p katkılı grafenin durumlar yoğunluğu

$\rho(\varepsilon)=\left\{\begin{array}{cc}2.17 \varepsilon+0.723 \mathrm{D} & \frac{\mathrm{D}}{3}<\varepsilon<\mathrm{D} \\ -2.17 \varepsilon+0.723 \mathrm{D} & -\mathrm{D}<\varepsilon \leq \frac{\mathrm{D}}{3}\end{array}\right.$

halindedir. Bu ifadelerde enerjinin çarpım katsayısı, Fermi hızının tersinin atomik birime dönüştürülmüş şeklidir.

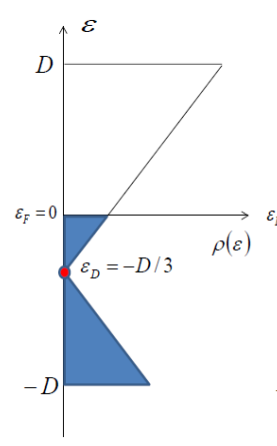

(a)

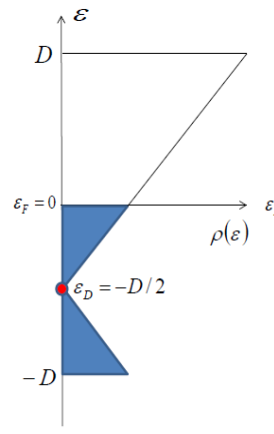

(b)

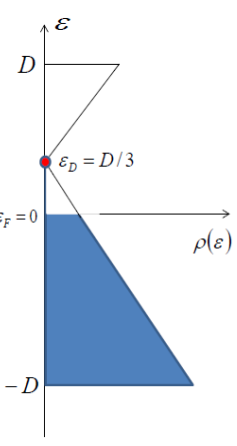

(c)
Şekil 1: Üç farklı grafen elektrodun durumlar yoğunluğu

Şekil 2'deki paneller kuantum noktacığının kesik enerji düzeyi son seviyeye çıkarıldıktan sonra Kondo rezonansının oluşmaya başladığı zaman ölçeğindeki anlık iletkenlik değerlerini farklı asimetri faktörleri için göstermektedir. Bu şekilde siyah (düz), kırmızı (nokta çizgili) ve yeşil (çizgili) eğriler (yukarıdan aşağıya) kuantum noktacığı son konumuna getirildikten sonra $\mathrm{T}=0.009 \Gamma_{\text {tot }}$ ortam sicaklığında ve $V=0.002 \Gamma_{t o t}$ voltaj değerinde anlık iletkenlik değerlerini zamana göre sırasıyla $\eta=0.85$, 0.90 ve 0.95 asimetri faktörleri için Kondo zaman ölçeğinde göstermektedir. Tepe, orta ve en alt paneller sırasıyla Şekil 1'deki a,b ve c panellerindeki durumlar yoğunluklarına karşılık gelmektedir. Burada açıkça görüleceği üzere n katkılı grafen için anlık iletkenlik karmaşık dalgalanmalar gösterirken, p katkılı grafen için ise basit sinüzoidal titreşimler görülmektedir. Bunun ötesinde hem $\mathrm{p}$ ve hem de $n$ 
katkılı elektrotlar için asimetri faktörü azaldıkça ve zaman ilerledikçe titreşim genliği azalmaktadır.
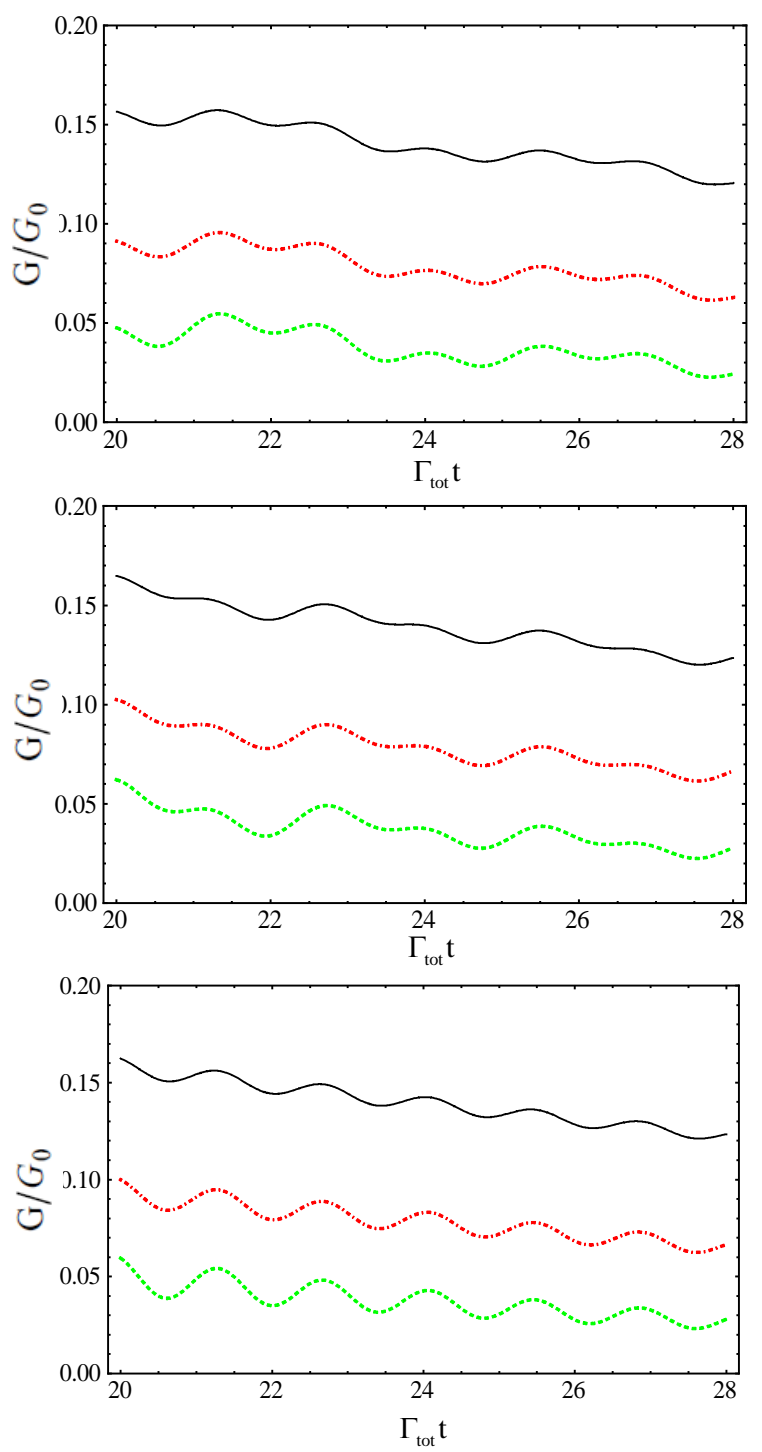

Şekil 2: Tepe, orta ve en alt paneller sırasıyla Şekil 1'deki a, b ve c panellerindeki elektrotlar için anlık iletkenlik değerlerini gösterir. Bütün panellerde yukarıdan aşağıya doğru asimetri faktörü $\eta=0.85$, 0.90 ve 0.95 .

Ortam sıcaklığının bu titreşimleri nasıl etkilediğini araştırmak için asimetri faktörü ๆ'yı sabit tutarak farklı sicaklıklarda hesaplamalarımızı tekrarladık. Elde ettiğimiz sonuçlar Şekil 3'te görülmektedir. Burada yeşil (çizgili), siyah (nokta çizgili), mavi (noktalı), kırmızı (düz), lacivert (düz) eğriler (yukarıdan aşağıya) kuantum noktacığı son konumuna getirildikten sonra $V=0.002 \Gamma_{t o t}$ voltaj değerinde ve $\eta=0.90$ asimetri faktöründe anlık iletkenlik değerlerini zamana göre sirasıyla $\mathrm{T}=0.0015 \Gamma_{t o t}, \quad \mathrm{~T}=0.0030 \Gamma_{t o t}, \quad \mathrm{~T}=0.0060 \Gamma_{t o t}$, $\mathrm{T}=0.0090 \Gamma_{\text {tot }}$ ve $\mathrm{T}=0.0150 \Gamma_{\text {tot }}$ ortam sicaklıkları için Kondo zaman ölçeğinde göstermektedir. Tepe, orta ve en alt paneller sırasıyla Şekil 1'deki a,b ve c panellerindeki durumlar yoğunluklarına karşılık gelmektedir. Buna bakarak bütün elektrotlar için ortam sıcaklığının artmasının titreşim genliğini baskıladığını görmekteyiz. Bilhassa ortam sıcaklığı Kondo sicaklığ 1 T $T_{K}$ 'nın çok üstüne çıktığ durumlarda titreşim genliklerinin tamamen yok olmak üzere olduğu bu şekilde açıkça göze çarpmaktadır.
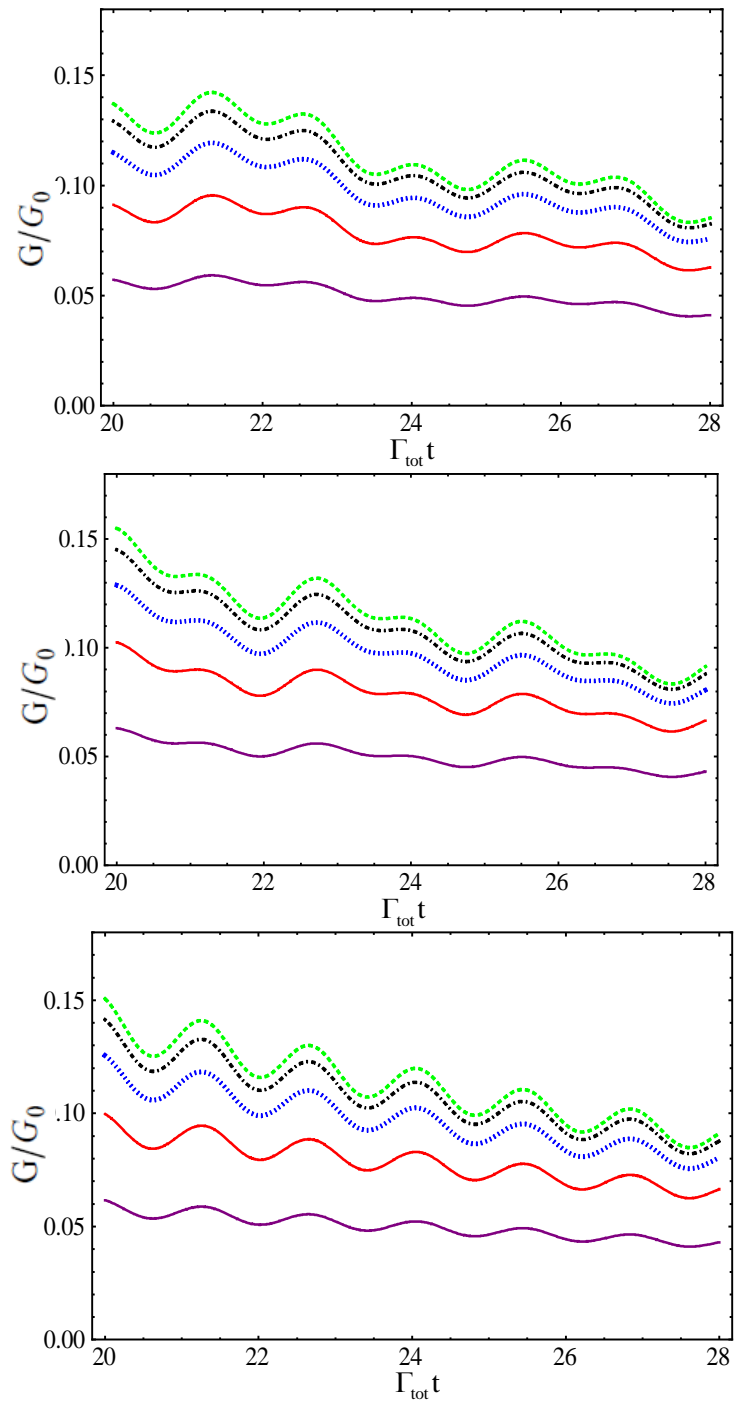

Şekil 3: Tepe, orta ve en alt paneller sırasıyla Şekil 1 'deki a, b ve c panellerindeki elektrotlar için anlık iletkenlik değerlerini gösterir. Bütün panellerde yukarıdan aşağı doğru $\mathrm{T}=0.0015 \Gamma_{\text {tot }}, \mathrm{T}=0.0030 \Gamma_{\text {tot }}$, $\mathrm{T}=0.0060 \Gamma_{\text {tot }}, \mathrm{T}=0.0090 \Gamma_{\text {tot }}$ ve $\mathrm{T}=0.0150 \Gamma_{\text {tot }}$.

$\mathrm{Bu}$ gözlemleri daha netleştirebilmek için bütün elektrot türleri için anlık iletkenlikte gözlemlenen titreşimlerde rol alan bütün titreşim frekanslarını tespit etmek amacıyla anlık iletkenlik verilerinin Fourier dönüşümünü gerçekleştirdik. Elde ettiğimiz sonuçlar Şekil 4'te sunulmuştur. Burada ilk göze çarpan husus n katkılı elektrotlar için iki farklı titreşim frekansı mevcutken, $\mathrm{p}$ katkılı elektrot için 
tek bir titreşim frekansının varlığıdır. Ayrıca, n katkılı elektrotlar için bulunan titreşim frekanslarının her iki elektrot için de Dirac noktasının Fermi düzeyine olan uzaklığı ve bant kenarının Fermi düzeyine uzaklığıyla orantılı olduğu görülmektedir. Nitekim, titreşim frekanslarının birbirine oranı, uzaklıkların oranıyla aynıdır. P katkılı elektrot için ise bulunan tek frekans bant kenarının Fermi düzeyine olan uzaklıkla orantılıdır. Bu şekilde not etmek istediğimiz bir diğer husus ise görülen eksi titreşim frekanslarıdır. Bunlar sinüzoidal bir fonksiyonun Fourier dönüşümünden kaynaklanmakta olup tamamen matematikseldir. Pozitif frekansların eksen etrafındaki simetrik değerleri olup ayrıca fiziksel bir anlam taşımamaktadırlar.
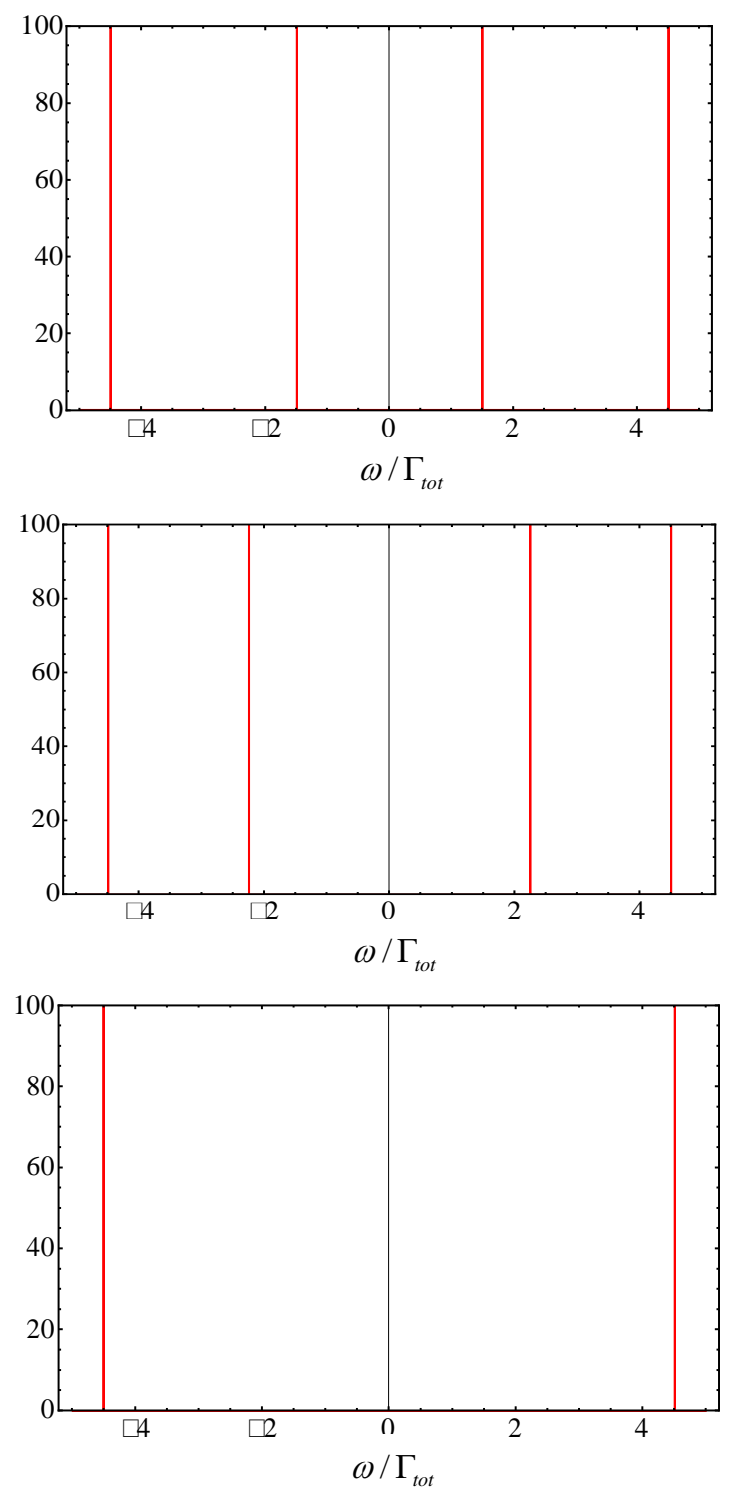

Şekil 4: Şekil 2 ve 3'te görülen anlık iletkenlik verilerinin Fourier dönüşümü. Tepe, orta ve en alt paneller sırasıyla Şekil 1'deki a,b ve c panellerindeki elektrotlara karşılık gelmektedir.
$\mathrm{Bu}$ sonuçlardan Fermi düzeyinde bulunan bir özelliğin elektrotların durumlar yoğunluğunda bulunan tekil noktalarla girişim oluşturduğu sonucu çıkarılabilir. Bu tekilliklere van Hove tekillikleri adı verilmekte ve bunların girişim yaptığı özelliğin de Fermi düzeyinde zamana bağlı olarak gelişmekte olan Kondo rezonansı olduğu daha önce mikroskopik bir modelle ortaya atılmıştı [6]. Buna göre asimetri faktörü azaldıkça titreşim genliğinin de giderek azalması Kondo rezonansının elektrotlarla denklem 13'e göre zıt fazlı girişime uğramasından kaynaklanmaktadır. Ayrıca, ortam sicaklığı Kondo sıcaklığının üstüne çıktığında titreşim genliğinin azalması da Kondo rezonansının giderek yok olmasından dolayıdır. Hem $p$ hem $n$ katkılı elektrotlar için zaman ilerledikçe titreşim genliğinin giderek sönümlenmesi ise Kondo rezonansının gelişimini tamamlamaya başlamasından kaynaklanmaktadır. Kondo rezonansının statik duruma geldiği durgunluk halinde titreşim yoktur. Özetlemek gerekirse, bu çalışmada değişik seviyelerde katkılı grafen elektrotlar kullanarak elde ettiğimiz sonuçlar bu ortaya konan modeli desteklemektedir.

\section{Değerlendirme}

Çalışmada elde edilen sonuçlar şöyle özetlenebilir:

- Katkılı grafen elektotlara asimetrik bağlanmış güçlü korelasyonlu bir kuantum noktacığın kesik düzeyi bir kapı voltajı yardımıyla aniden elektotların Fermi düzeyine yakın bir seviyeye getirildiğinde devrede gözlenen anlık iletkenlik karmaşık titreşimler gösterir.

- $\mathrm{Bu}$ titreşimlerin genliği ortam sıcaklığı Kondo sıcaklığının üstüne çıktıkça ve asimetri faktörü azaldıkça bütün elektrot türleri için giderek azalmaktadır.

- Kondo ölçeğinde zaman ilerledikçe yine bütün elektrot türleri için titreşimler giderek sönümlenmekte ve durgunluk durumuna ulaşıldığında tamamen yok olmaktadır.

- Fourier dönüşümü yoluyla bu titreşimlerin analizi yapıldığında, titreşimlerin $\mathrm{n}$ katkılı elektrotlarda Dirac noktası ve bant kenarının elektrodun Fermi düzeyine uzaklığıyla orantılı iki farklı frekansta gerçekleşirken, p katkılı elektrotta ise bant kenarının Fermi düzeyine uzaklığıyla orantılı tek bir frekansta gerçekleştiği görülmüştür.

- Bu gözlemleri, daha önce ortaya atılan Fermi düzeyinde Kondo zaman ölçeğinde gelişmekte olan dinamik Kondo rezonansinın elektrotlardaki van Hove tekillikleriyle zit fazda girişime uğradığını öne süren mikroskopik bir teoriyle bağdaştırdık. 
Sonuç olarak, gelecekte kullanılabilecek grafen tabanlı tranzistörlerin ani açılış davranışlarına 1 şı tutmuş olduk ve bu tür tranzistörlerin üretimi ve

\section{Kaynaklar}

[1] Nordlander, P., Pustilnik, M., Meir, Y., Wingreen, N.S. ve Langreth, D.C., How long does it take for the Kondo effect to develop?, Physical Review Letters, 83, 4, 808-811, (1999).

[2] Plihal, M., Langreth, D.C. ve Nordlander, P., Kondo time scales for quantum dots: Response to pulsed bias potentials, Physical Review B, 61, 20, R13341-R13344, (2000).

[3] Schiller, A. ve Hershfield, S., Out-ofequilibrium Kondo effect: Response to pulsed fields, Physical Review B, 62, 24, R16271R16274, (2000).

[4] Plihal, M., Langreth, D.C. ve Nordlander, P., Transient currents and universal timescales for a fully time-dependent quantum dot in the Kondo regime, Physical Review B, 71, 16, 165321, (2005).

[5] Anders, F.B. ve Schiller, A., Real time dynamics in quantum-impurity systems: A time-dependent numerical renormalizationgroup approach, Physical Review Letters, 95, 19, 196801, (2005).

[6] Goker, A., Friedman, B.A. ve Nordlander P., Transient current in a quantum dot asymmetrically coupled to metallic leads, Journal of Physics: Condensed Matter, 19, 37, 376206, (2007).

[7] Novoselov, K.S., Geim, A.K. , Morozov, S.V., Jiang, D., Zhang, Y., Dubonos, S.V., Grigorieva, I.V. ve Firsov, A.A., Electric field effect in atomically thin carbon films, Science, 306, 5696, 666-669, (2004).

[8] Nair, R. R., Blake, P., Grigorenko, A. N., Novoselov, K. S., Booth, T. J., Stauber, T., Peres, N.M.R. ve Geim, A.K., Fine structure constant defines visual transparency of graphene, Science, 320, 5881, 1308, (2008).

[9] Lee, C., Wei, X., Kysar, J.W. ve Hone, J., Measurement of the elastic properties and karakterizasyonu için yol gösterici sonuçlar elde etmiş bulunmaktayı.

intrinsic strength of monolayer graphene, Science, 321, 5887, 385-388, (2008).

[10] Balandin, A.A., Thermal properties of graphene and nanostructured carbon materials, Nature Materials, 10, 8, 569-581, (2011).

[11] Anderson, P.W., Localized magnetic states in metals, Physical Review, 124, 41-53, (1961).

[12] Kadanoff, L.P. ve Baym, G., Quantum statistical mechanics, New York, W.A. Benjamin, (1976).

[13] Langreth, D.C., Linear and nonlinear response theory with applications in J.T. Devreese and V.E. van Doren, Linear and nonlinear electron transport in solids, Plenum Press, 3-32, New York NY, (1976).

[14] Coleman, P., New approach to the mixed valence problem, Physical Review B, 29, 6, 3035-3044, (1984).

[15] Langreth, D.C. ve Nordlander, P., Derivation of a master equation for charge-transfer processes in atom-surface collisions, Physical Review B, 43, 4, 2541-2557, (1991).

[16] Shao, H.X., Langreth, D.C. ve Nordlander, P., Many-body theory for charge-transfer in atom-surface collisions, Physical Review B, 49, 19, 13929-13947, (1994).

[17] Langreth, D.C. ve Nordlander, P., Derivation of a master equation for charge-transfer processes in atom-surface collisions, Physical Review B, 43, 4, 2541-2557, (1991).

[18] Kondo, J., Resistance minimum in dilute magnetic alloys, Progress in Theoretical Physics, 32, 1, 37-49, (1964).

[19] Izmaylov, A.F., Goker, A., Friedman, B.A. ve Nordlander, P., Transient current in a quantum dot subject to a change in coupling to its leads, Journal of Physics: Condensed Matter, 18, 39, 8995-9006, (2006). 